\title{
Tendência temporal da incidência da AIDS em pessoas com 50 anos ou mais no Brasil $^{*}$
}

Temporal trend in the incidence of AIDS in people aged 50 and over in Brazil

Tendencia temporal de la incidencia del sida en personas de 50 años y más en Brasil

\begin{abstract}
Aenne Zandonadi Rodrigues Santana ${ }^{\mathrm{I}}$, Annelita Almeida Oliveira Reiners ${ }^{\mathrm{II}}$, Rosemeiry Capriata de Souza Azevedo ${ }^{\text {III }}$, Janderson Diego Pimenta da Silva ${ }^{\mathrm{IV}}$, Amanda Cristina de Souza Andradev , Priscila Aguiar Mendes ${ }^{\mathrm{VI}}$
\end{abstract}

\begin{abstract}
Resumo: Objetivo: analisar a tendência temporal da incidência da Síndrome da Imunodeficiência Adquirida (AIDS) em pessoas com 50 anos ou mais no Brasil e suas regiões. Método: estudo ecológico misto dos casos notificados de AIDS em pessoas de 50 anos ou mais entre 2007 e 2017, no Brasil e regiões. Os dados foram extraídos do Sistema Nacional de Agravos e Notificação e analisados pelo método Prais-Winsten. Resultados: a tendência temporal da incidência de AIDS em pessoas com 50 anos ou mais foi estável no Brasil e nas regiões Sudeste, Sul e Centro-Oeste (variação percentual anual: 10,7; 4,9; 8,4 e 12,2, respectivamente); nas regiões Norte e Nordeste, foi crescente (variação percentual anual: 16,6 e 20,6, respectivamente). Conclusão: houve estabilidade da incidência de AIDS no Brasil e nas regiões Sudeste, Sul e Centro-Oeste, como também aumento da incidência da doença nas regiões Norte e Nordeste, na população estudada.

Descritores: Síndrome de Imunodeficiência Adquirida; Estudos Ecológicos; Pessoa de Meia-idade; Idoso; Notificação de Doenças
\end{abstract}

\footnotetext{
${ }^{\text {I }}$ Enfermeira, mestre em enfermagem, Faculdade de Enfermagem/Universidade Federal de Mato Grosso, Cuiabá, Mato, Brasil. E-mail: aennesantana@gmail.com, Orcid: https://orcid.org/0000-0003-2711-0384.

II Enfermeira, doutora em enfermagem, Faculdade de Enfermagem/Universidade Federal de Mato Grosso, Cuiabá, Mato Grosso, Brasil. E-mail: annereiners.ar@gmail.com, Orcid: http://orcid.org/0000-0002-5699-8215.

III Enfermeira, doutora em enfermagem, Faculdade de Enfermagem/Universidade Federal de Mato Grosso, Cuiabá, Mato Grosso, Brasil. E-mail: rosemeirycapriataazevedo@gmail.com, Orcid: http://orcid.org/0000-0001-7986-5768.

IV Enfermeiro, mestre em enfermagem, Faculdade de Enfermagem/Universidade Federal de Mato Grosso, Cuiabá, Mato Grosso, Brasil. E-mail: jandersonpimenta@hotmail.com, Orcid: https://orcid.org/0000-0001-6024-7134.

V Estatística, doutora em saúde pública, Instituto de Saúde Coletiva/Universidade Federal de Mato Grosso, Cuiabá, Mato Grosso, Brasil. E-mail: amandasouza_est@yahoo.com.br, Orcid: https://orcid.org/0000-0002-3366-4423.

VI Enfermeira, mestre em enfermagem, Faculdade de Enfermagem/Universidade Federal de Mato Grosso, Cuiabá, Mato Grosso, Brasil. E-mail: prih.mendes@gmail.com, Orcid: https://orcid.org/0000-0002-0752-091X.
}

* Extraído da dissertação “Aids em pessoas de 50 anos e mais no Brasil no período de 2007 - 2017: tendência e características”, Programa de Pós-Graduação em Enfermagem, Universidade Federal de Mato Grosso, 2020. 
Abstract: Objective: to analyze the temporal trend of the incidence of Acquired Immunodeficiency Syndrome (AIDS) in people aged 50 years or more in Brazil and its regions. Method: a mixed ecological study of reported cases of AIDS in people aged 50 years or more between 2007 and 2017, in Brazil and regions. Data were extracted from the National System of Diseases and Notification and analyzed using the Prais-Winsten method. Results: the temporal trend of the incidence of AIDS in people aged 50 years or more was stable in Brazil and the Southeast, South, and Midwest regions (annual percentage variation: 10.7; 4.9; 8.4 and 12.2, respectively); in the North and Northeast regions, it was increasing (annual percentage variation: 16.6 and 20.6, respectively). Conclusion: the incidence of AIDS in Brazil and the Southeast, South and Midwest regions were stable, as well as an increase in the incidence of the disease in the North and Northeast regions, in the population studied.

Descriptors: Acquired Immunodeficiency Syndrome; Ecological Studies; Middle-aged; Aged; Disease Notification

Resumen: Objetivo: analizar la tendencia temporal de la incidencia del Síndrome de Inmunodeficiencia Adquirida (SIDA) en personas de 50 años o más en Brasil y sus regiones. Método: estudio ecológico mixto de casos notificados de sida en personas de 50 años o más entre 2007 y 2017, en Brasil y regiones. Los datos fueron extraídos del Sistema Nacional de Enfermedades y Notificación y se analizaron mediante el método Prais-Winsten. Resultados: la tendencia temporal de la incidencia de SIDA en personas de 50 años o más fue estable en Brasil y en las regiones Sudeste, Sur y Centro-Oeste (variación porcentual anual: 10,7; 4,9; 8,4 y 12,2, respectivamente); y en las regiones Norte y Nordeste aumentó (variación porcentual anual: 16,6 y 20,6, respectivamente). Conclusión: hubo estabilidad en la incidencia de sida en Brasil y en las regiones Sudeste, Sur y Centro-Oeste, así como un aumento en la incidencia de la enfermedad en las regiones Norte y Nordeste, en la población estudiada.

Descriptores: Síndrome de Inmunodeficiencia Adquirida; Estudios Ecológicos; Persona de Mediana Edad; Anciano; Notificación de Enfermedades

\section{Introdução}

Mesmo após quase 40 anos de seu surgimento, a Síndrome da Imunodeficiência Adquirida (AIDS) causada pelo Vírus da Imunodeficiência Humana (HIV) é considerada um problema de saúde pública. Até o fim de 2018, cerca de 44 milhões de pessoas viviam com o HIV no mundo e 1,1 milhão de óbitos por causas relacionadas à AIDS ocorreram. ${ }^{1}$ No Brasil, em 2018, foram notificados 43.941 casos novos de HIV e 37.161 de AIDS. ${ }^{2}$ Nesse mesmo ano, a taxa de detecção de AIDS na população em geral foi de 17,8/100.000 habitantes. ${ }^{2}$

Desde o surgimento do HIV e da AIDS, o perfil epidemiológico das pessoas que adquirem e vivem com HIV mudou. No início, era comum em jovens, homossexuais, residentes em grandes cidades e pessoas com maior nível socioeconômico. Na década de 90, gradualmente chegou a cidades menores e atingiu outros segmentos populacionais, passando a ser 
3 | Santana AZR, Reiners AAO, Azevedo RCS, Silva JDP, Andrade ACS, Mendes PA

diagnosticado também em pessoas com baixo nível socioeconômico, heterossexuais e em mulheres. Nas décadas seguintes, observou-se aumento da incidência tanto do vírus quanto da doença em homossexuais e homens e em pessoas de maior idade, como as de 50 anos ou mais. ${ }^{3-4}$

No Brasil, a média anual de registro de novos casos de AIDS desde 2013 foi de 40 mil, dos quais cerca de $20 \%$ foram de pessoas na faixa etária de 50 anos ou mais. ${ }^{2}$ Para essa população e suas famílias, o ônus do HIV/AIDS é significativo em razão das inúmeras alterações físicas, psicológicas e sociais, como aumento de chances de infecções oportunistas, hospitalizações, limitações funcionais, estresse, depressão, discriminação e isolamento social. ${ }^{4}$ Uma hipótese para esse aumento de casos é o prolongamento da vida sexual desses indivíduos aliado a práticas sexuais desprotegidas ou ao fato de que o uso de Terapia Antirretroviral (TARV) tenha possibilitado o envelhecimento das pessoas que vivem com HIV (PVHIV). ${ }^{4-5}$

Esse aumento gradativo de PVHIV no mundo e no Brasil tem dado origem a diversos tipos de estudos sobre HIV e AIDS na população adulta, entre eles várias pesquisas com o objetivo de investigar a distribuição no tempo da incidência, prevalência e/ou mortalidade. ${ }^{6-9}$ Entretanto, poucos estudos de tendência temporal do HIV ou da AIDS em pessoas com 50 anos ou mais foram encontrados na literatura. Na China, verificou-se aumento significativo da incidência de HIV e AIDS nessa população entre 2004 e $2014 .{ }^{10}$ Em Botsuana, na série temporal de 2004 a 2013, encontrou-se tendência crescente da prevalência do HIV em pessoas com 50 ou mais. ${ }^{11}$

No Brasil, a maioria das pesquisas com objetivo de estimar tendências da AIDS em pessoas com 50 anos ou mais utilizou como unidade de análise estados ou municípios e em todas se observou aumento da incidência da doença nessa população. ${ }^{12-16}$ Estudos com casos de indivíduos nesta faixa etária, com dados consolidados nacionalmente, são escassos. Um deles avaliou a tendência temporal da AIDS no Brasil no período de $1980-2008$ em pessoas com mais de 50 anos, porém comparando com outras idades. ${ }^{17}$ Outro teve objetivo e nível de desagregação semelhantes, no entanto utilizou a mortalidade como indicador e o período foi de $2000-2011 .^{8}$ 
Tendência temporal da incidência da AIDS em pessoas com 50 anos ou mais no Brasil | 4

Considerando a mudança no perfil epidemiológico da AIDS ao longo dos anos, o prolongamento da vida sexual da população, o envelhecimento das PVHIV, o ônus do HIV e a escassez de estudos de tendência temporal da incidência da AIDS em pessoas com 50 anos ou mais, tem-se como questão de pesquisa: qual a tendência da AIDS em pessoas de 50 anos ou mais no Brasil e suas regiões? Para responder a esse questionamento, teve-se como objetivo analisar a tendência temporal da incidência da AIDS em pessoas com 50 anos ou mais no Brasil e suas regiões.

\section{Método}

Trata-se de estudo ecológico misto dos coeficientes de incidência de AIDS em pessoas de 50 anos ou mais no Brasil e suas regiões geográficas, no período de 2007 a 2017.

O estudo foi realizado com os dados do Sistema Nacional de Agravos e Notificação (SINAN), que reúne informações sobre todos os casos de doenças de notificação compulsória e estão disponíveis no Departamento de Informática do Sistema Único de Saúde (DATASUS). Os dados populacionais foram obtidos por meio da projeção da população das Unidades da Federação por sexo e grupos de idade: 2000 a 2030 do Instituto Brasileiro de Geografia e Estatística (IBGE). ${ }^{18}$ A população foi composta por todos os casos notificados de AIDS em pessoas de 50 anos ou mais. Essa faixa etária foi escolhida considerando o rápido processo de envelhecimento populacional ocorrido no Brasil nos últimos anos e a mudança da distribuição etária da epidemia de HIV no mundo. ${ }^{4}$

As informações foram coletadas no mês de outubro de 2019. Incluíram-se todos os casos de AIDS em pessoas de 50 anos ou mais notificados no período analisado. A notificação no SINAN segue os critérios de definição de AIDS em adultos CDC-adaptado e/ou Rio de Janeiro/Caracas, estabelecidos pelo Ministério da Saúde (MS). O período do estudo foi escolhido considerando a disponibilidade de informações completas no banco de dados utilizado. 
5 | Santana AZR, Reiners AAO, Azevedo RCS, Silva JDP, Andrade ACS, Mendes PA

Os dados foram gerados em arquivo Comma Separated Values (CSV) considerando as seguintes variáveis de interesse: sexo (masculino, feminino), cor/raça (branca, preta, amarela, parda, indígena, ignorado), escolaridade (analfabeto, $1^{\underline{a}}$ a $4^{\underline{a}}$ série incompleta, $4^{\underline{a}}$ série completa, $5^{\underline{a}}$ a $8^{\underline{a}}$ série incompleta, fundamental completo, médio incompleto, médio completo, superior incompleto, superior completo), faixa etária (50-59, 60-69, 70-79, 80 e mais), categoria de exposição (homossexual, bissexual, heterossexual, usuário de drogas injetáveis, hemofílico, transfusão, acidente biológico e ignorado) e ano (2007, 2008, 2009, 2010, 2011, 2012, 2013, 2014, 2015, 2016 e 2017).

Em seguida, os dados foram exportados para planilha eletrônica do Microsoft Office Excel e organizados. As características dos casos de AIDS em pessoas de 50 anos ou mais foram analisadas segundo Brasil e região de notificação por meio de frequência absoluta e relativa.

Os coeficientes de incidência anuais da AIDS foram calculados por 100.000 habitantes. Posteriormente, realizou-se a padronização direta dos coeficientes de incidência ajustada por idade, usando como referência a população brasileira a partir de projeções intercensitárias (2000 - 2030) disponíveis no DATASUS, com o objetivo de anular o efeito da distribuição demográfica desigual da população.

A análise da tendência foi realizada para todo o Brasil e regiões geográficas por meio do modelo de regressão linear generalizada pelo método de Prais-Winsten. A variável dependente foi o logaritmo dos coeficientes e a variável independente os anos da série temporal. A variação percentual anual (VPA) foi calculada pela fórmula $-1+10 b=\Delta$, e para o intervalo de confiança (IC) $\Delta_{I C 95 \%}=-1+10^{\left(b \pm t^{*} s e\right)}$ em que “ $b$ ” corresponde à taxa de crescimento anual. Os valores de “ $b$ ” e erro padrão (se) foram extraídos da análise de regressão e o valor de " $t$ " fornecido pela tabela da distribuição $t$ de Student.

A tendência crescente, decrescente ou estável foi expressa como VPA, com os respectivos IC (95\%). Considerou-se estável (se p>0,05), decrescente (se $p<0,05$ e coeficiente de regressão negativo) e crescente (se $\mathrm{p}<0,05$ e coeficiente de regressão positivo). Para a análise de tendência, 
Tendência temporal da incidência da AIDS em pessoas com 50 anos ou mais no Brasil | 6

utilizou-se o software Stata, versão 12.0. Apesar de o estudo ter sido realizado com dados secundários, cumpriram-se todos os preceitos éticos estabelecidos na Resolução 466/2012 do Conselho Nacional de Saúde.

\section{Resultados}

No Brasil, no período de 2007 a 2017, notificaram-se 49.188 casos de AIDS em pessoas de 50 anos ou mais de idade. O maior número de casos foi registrado na faixa etária entre 50 e 59 $\operatorname{anos}(35.599 ; 72,4 \%)$, no sexo masculino $(30.619 ; 62,2 \%)$, na raça/cor branca $(23.480 ; 47,7 \%)$, na escolaridade de $5^{\mathbf{a}}$ a $8^{\mathrm{a}}$ série incompleta $(8.142 ; 22,5 \%)$ e na categoria de exposição heterossexual $(32.678 ; 66,4 \%)$ (Tabela 1$)$.

Quanto à distribuição dos casos de AIDS na população estudada segundo as regiões brasileiras, o maior número de casos foi registrado na região Sudeste $(20.898 ; 42,5 \%)$, seguida da região Sul $(13.230 ; 26,9 \%)$. Houve predomínio de casos no sexo masculino nas regiões Norte $(66,1 \%)$, Nordeste $(66,2 \%)$, Sudeste $(62,3 \%)$ e Centro-Oeste $(61,2 \%)$. A raça/cor parda foi mais frequente nas regiões Norte $(74,2 \%)$, Nordeste $(65,5 \%)$ e Centro-Oeste $(48,7 \%)$. Nas regiões Sudeste $(49,7 \%)$ e Sul (77\%) houve predomínio da raça/cor branca. A escolaridade de $5^{\mathbf{a}}$ a $8^{\mathbf{a}}$ série incompleta foi mais frequente nas regiões Norte $(22,4 \%)$, Sudeste $(20,5 \%)$, Sul (28\%) e CentroOeste (22,4\%). Na região Nordeste $(19,8 \%)$, a escolaridade de $1^{\text {a }}$ a $4^{\text {a }}$ série incompleta foi mais frequente. A faixa etária de 50 a 59 anos e a categoria de exposição heterossexual representaram a maioria dos casos em todas as regiões brasileiras (Tabela 1). 
7 | Santana AZR, Reiners AAO, Azevedo RCS, Silva JDP, Andrade ACS, Mendes PA

Tabela 1 - Distribuição dos casos de AIDS notificados entre pessoas de 50 anos e mais, no Brasil e suas regiões segundo variáveis sociodemográficas. Brasil, 2007-2017.

\begin{tabular}{|c|c|c|c|c|c|c|}
\hline \multirow[t]{2}{*}{ Variáveis } & Brasil & Norte & Nordeste & Sudeste & Sul & $\begin{array}{c}\text { Centro- } \\
\text { Oeste }\end{array}$ \\
\hline & $\%(n)$ & $\%(n)$ & $\%(n)$ & $\%(n)$ & $\%(\mathrm{n})$ & $\%(\mathrm{n})$ \\
\hline \multicolumn{7}{|l|}{ Sexo } \\
\hline Total & 49.188 & 3.129 & 8.501 & 20.898 & 13.230 & 3.430 \\
\hline Masculino & $62,2(30.619)$ & $66,1(2.067)$ & $66,2(5.631)$ & $62,3(13.013)$ & $59,0(7.808)$ & $61,2(2.100)$ \\
\hline Feminino & 37,8 (18.569) & $33,9(1.062)$ & $33,8(2.870)$ & 37,7 (7.885) & $41,0(5.422)$ & $38,8(1.330)$ \\
\hline \multicolumn{7}{|l|}{ Faixa etária } \\
\hline Total & 49.188 & 3.129 & 8.501 & 20.898 & 13.230 & 3.430 \\
\hline $50-59$ & 72,4 (35.599) & $73,6(2.304)$ & $72,3(6.147)$ & 72,7 (15.193) & $71,9(9.510)$ & $71,3(2.445)$ \\
\hline $60-69$ & $22,4(11.035)$ & $20,8(651)$ & $22,6(1.918)$ & $22,2(4.634)$ & $23,2(3.075)$ & $22,1(757)$ \\
\hline $70-79$ & $4,5(2.214)$ & $4,5(141)$ & $4,2(359)$ & $4,5(936)$ & $4,4(578)$ & $5,8(200)$ \\
\hline 80 e mais & $0,7(340)$ & $1,1(33)$ & $0,9(77)$ & $0,6(135)$ & $0,5(67)$ & $0,8(28)$ \\
\hline \multicolumn{7}{|l|}{ Raça/cor } \\
\hline Total & 49.188 & 3129 & 8.501 & 20.898 & 13.230 & 3.430 \\
\hline Branca & $47,7(23.480)$ & $13,5(421)$ & $15,8(1.342)$ & 49,7 (10.381) & 77,0 (10.182) & $33,6(1.154)$ \\
\hline Preta & $10,2(5.040)$ & $5,2(164)$ & $10,3(875)$ & $12,3(2.562)$ & $8,8(1.169)$ & $7,9(270)$ \\
\hline Amarela & $0,5(236)$ & $0,4(14)$ & $0,3(25)$ & $0,5(114)$ & $0,4(49)$ & $1,0(34)$ \\
\hline Parda & $33,6(16.520)$ & $74,2(2.321)$ & $65,5(5.565)$ & $27,0(5.650)$ & 9,9 (1.313) & 48,7 (1.671) \\
\hline Indígena & $0,3(124)$ & $0,9(29)$ & $0,2(18)$ & $0,1(27)$ & $0,2(23)$ & $0,8(27)$ \\
\hline Ignorado & 7,7 (3.788) & $5,8(180)$ & $8,0(676)$ & $10,4(2.164)$ & 3,7 (494) & $8,0(274)$ \\
\hline \multicolumn{7}{|l|}{ Escolaridade } \\
\hline Total & 36.132 & 2.249 & 6.020 & 14.461 & 10.938 & 2.464 \\
\hline Analfabeto & $6,6(2.374)$ & $10,7(240)$ & $14,2(856)$ & $4,5(650)$ & $3,9(426)$ & $8,2(202)$ \\
\hline $\begin{array}{l}1^{\text {a }} \text { a } 4^{\mathrm{a}} \text { série } \\
\text { incompleta }\end{array}$ & $16,8(6.061)$ & $20,4(458)$ & $19,8(1.191)$ & $15,6(2.261)$ & $15,2(1.660)$ & $19,9(491)$ \\
\hline $\begin{array}{l}4^{\mathrm{a}} \text { série } \\
\text { completa }\end{array}$ & $11,6(4.186)$ & $10,7(240)$ & $9,5(572)$ & $12,0(1.741)$ & 12,8 (1.399) & $9,5(234)$ \\
\hline $\begin{array}{l}5^{\mathrm{a}} \text { a } 8^{\mathrm{a}} \text { série } \\
\text { incompleta }\end{array}$ & $22,5(8.142)$ & $22,4(504)$ & 17,7 (1.064) & $20,5(2.960)$ & $28,0(3.061)$ & $22,4(553)$ \\
\hline $\begin{array}{l}\text { Fundamental } \\
\text { completo }\end{array}$ & $12,9(4.661)$ & $8,0(181)$ & $10,4(624)$ & $14,5(2.104)$ & $13,6(1.490)$ & $10,6(262)$ \\
\hline $\begin{array}{l}\text { Médio } \\
\text { incompleto }\end{array}$ & $5,7(2.044)$ & 4,6 (103) & $4,5(272)$ & $5,9(848)$ & $6,1(669)$ & $6,2(152)$ \\
\hline $\begin{array}{l}\text { Médio } \\
\text { completo }\end{array}$ & $14,3(5.172)$ & $14,9(335)$ & 15,8 (949) & $15,5(2.239)$ & $12,3(1.350)$ & $12,1(299)$ \\
\hline $\begin{array}{l}\text { Superior } \\
\text { incompleto }\end{array}$ & $1,9(676)$ & $1,8(40)$ & $1,4(86)$ & $2,0(292)$ & $1,9(209)$ & $2,0(49)$ \\
\hline $\begin{array}{l}\text { Superior } \\
\text { completo }\end{array}$ & $7,8(2.816)$ & $6,6(148)$ & $6,7(406)$ & $9,4(1.366)$ & $6,2(674)$ & $9,0(222)$ \\
\hline Categoria de & & & & & & \\
\hline exposição & & & & & & \\
\hline
\end{tabular}


Tendência temporal da incidência da AIDS em pessoas com 50 anos ou mais no Brasil | 8

\begin{tabular}{|c|c|c|c|c|c|c|}
\hline Total & 49.219 & 3.133 & 8.488 & 20.912 & 13.252 & 3.434 \\
\hline Homossexual & $7,7(3.811)$ & $7,0(218)$ & $8,8(743)$ & 9,1 (1.901) & $5,6(744)$ & $6,0(205)$ \\
\hline Bissexual & $4,1(2.029)$ & $4,1(130)$ & $4,6(387)$ & $4,7(990)$ & $3,1(405)$ & $3,4(117)$ \\
\hline Heterossexual & $66,4(32.678)$ & 75,8 (2.374) & $63,1(5.354)$ & $60,4(12.622)$ & $74,0(9.812)$ & $73,3(2.516)$ \\
\hline $\mathrm{UDI}^{*}$ & $1,9(938)$ & $0,8(26)$ & $0,9(76)$ & $2,1(445)$ & 2,7 (359) & $0,9(32)$ \\
\hline Hemofílico & $0,0(11)$ & $0,1(2)$ & $0,1(5)$ & $0,0(3)$ & $0,0(1)$ & $0,0(0)$ \\
\hline Transfusão & $0,1(25)$ & $0,0(0)$ & $0,0(0)$ & $0,1(14)$ & $0,1(9)$ & $0,1(2)$ \\
\hline $\begin{array}{l}\text { Acidente } \\
\text { biológico }\end{array}$ & $0,0(3)$ & $0,0(0)$ & $0,0(0)$ & $0,0(1)$ & $0,0(2)$ & $0,0(0)$ \\
\hline Ignorado & 19,8 (9.724) & 12,2 (383) & $22,7(1.923)$ & 23,6 (4.936) & $14,5(1.920)$ & $16,3(562)$ \\
\hline
\end{tabular}

Fonte: Sistema de Informação de Agravos de Notificação (SINAN/AIDS). Departamento de Informática do SUS. ${ }^{*}$ UDI: usuários de drogas injetáveis

Quando analisados conjuntamente, os coeficientes de incidência por AIDS apresentaram estabilidade com coeficiente médio de 10,9/100.000 habitantes. Quando analisadas por regiões, observou-se tendência crescente nas regiões Norte e Nordeste e de estabilidade nas demais regiões do país (Tabela 2).

Tabela 2 - Número e porcentagem de casos de AIDS notificados, coeficiente médio por 100.000 habitantes e tendência dos coeficientes de incidência de AIDS em pessoas de 50 anos e mais segundo regiões. Brasil, 2007-2017

\begin{tabular}{ccccccc}
\hline \multirow{2}{*}{ Região } & \multicolumn{2}{c}{ Casos } & $\begin{array}{c}\text { Coeficiente } \\
\text { médio }\end{array}$ & VPA $^{\ddagger}$ & IC95\% & Interpretação \\
\cline { 2 - 3 } Norte & 3.129 & 6,4 & 11,9 & 16,6 & 4,$2 ; 30,5$ & Crescente \\
Nordeste & 8.501 & 17,3 & 7,9 & 20,6 & 5,$1 ; 38,3$ & Crescente \\
Sudeste & 20.898 & 42,5 & 9,9 & 4,9 & $-5,3 ; 16,2$ & Estável \\
Sul & 13.230 & 26,8 & 17,8 & 8,4 & $-7,1 ; 26,5$ & Estável \\
Centro-Oeste & 3.430 & 7,0 & 11,2 & 12,2 & $-5,3 ; 33,0$ & Estável \\
Brasil & 49.188 & 100,0 & 10,9 & 10,7 & $-3,1 ; 26,4$ & Estável \\
\hline
\end{tabular}

'Coeficientes padronizados pela população brasileira. "Variação percentual anual. ${ }^{\S}$ Intervalo de confiança da VPA.

O aumento dos coeficientes de incidência de AIDS entre 2007 e 2017 nas regiões Norte e Nordeste, como a estabilidade dos coeficientes de incidência de AIDS no Brasil e regiões Sudeste, Sul e Centro-Oeste, está representado na Figura 1. 
9 | Santana AZR, Reiners AAO, Azevedo RCS, Silva JDP, Andrade ACS, Mendes PA

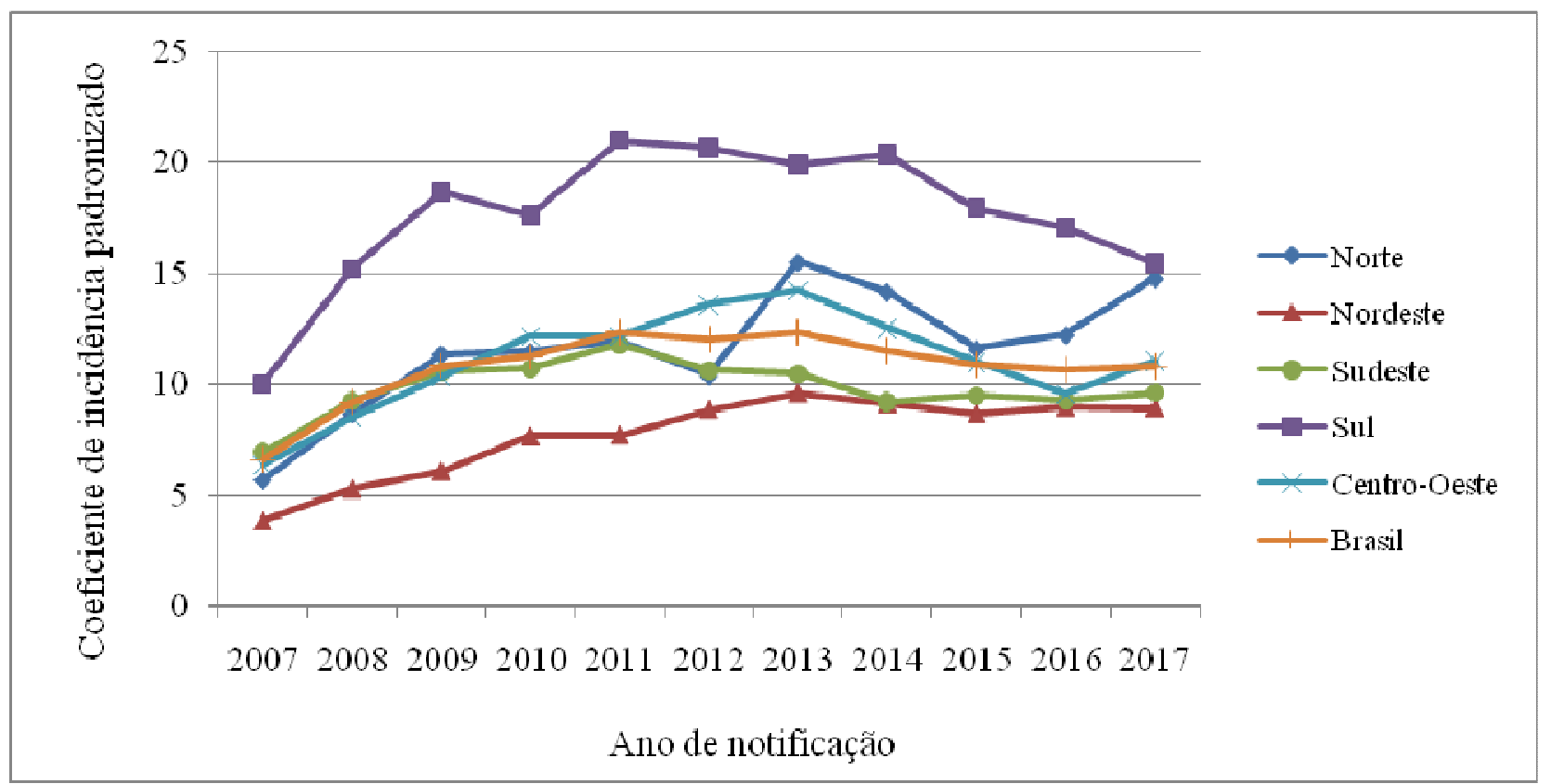

Nota: Coeficientes padronizados pela população do Brasil.

Figura 1 - Série temporal dos coeficientes de incidência de AIDS segundo regiões brasileiras. Brasil, 2007-2017.

\section{Discussão}

O principal achado deste estudo foi a tendência estável dos coeficientes de incidência de AIDS em pessoas de 50 anos ou mais no Brasil. É provável que a tendência de estabilidade encontrada esteja relacionada aos avanços ocorridos nos serviços de saúde e nas políticas públicas no que diz respeito ao diagnóstico, tratamento e monitoramento do HIV/AIDS, principalmente a introdução da TARV. ${ }^{19}$ Isso contribuiu para o controle da infecção, redução da mortalidade e aumento da expectativa de vida, tornando a AIDS uma doença crônica. ${ }^{4}$

Além disso, essa tendência pode ter sido influenciada pela diminuição na taxa de detecção de AIDS na população em geral. Em 2012, a taxa era de 21,4/100.000 habitantes e passou para 17,8/100.000 habitantes em 2018, representando decréscimo de $16,8 \%{ }^{2}$ 
Tendência temporal da incidência da AIDS em pessoas com 50 anos ou mais no Brasil | 10

A análise das tendências temporais dos coeficientes de incidência da AIDS por regiões neste estudo demonstra diferentes padrões. Na literatura, não foram encontradas pesquisas que analisaram a tendência temporal da AIDS em pessoas de 50 anos ou mais com base regional, o que dificulta a comparação de resultados. Entretanto, estudos realizados em estados do Nordeste ${ }^{12-13,15}$ e do Norte $^{16}$ mostram que houve aumento de casos de AIDS em adultos com 50 anos ou mais.

$\mathrm{Na}$ Paraíba, identificou-se tendência crescente da incidência em torno de $232 \%$ em pouco mais de 10 anos. Enquanto que na população em geral, houve pouca variação, com crescimento de $5 \% .^{13}$ No Ceará, também foi encontrada tendência crescente, principalmente em pessoas na faixa etária de 60 a 69 anos. ${ }^{12} \mathrm{Na}$ região Norte, estudo realizado no estado de Rondônia verificou aumento de $440 \%$ na incidência de HIV/AIDS em pessoas com 50 anos e mais, no período de 2000 a 2011. ${ }^{16} \mathrm{O}$ aumento observado na tendência nessas regiões pode ser decorrente da melhora do acesso aos serviços de saúde e dos sistemas de notificação, investigação e processamento dos dados ao longo dos anos, que pode ter contribuído para mostrar casos que anteriormente não eram notificados. ${ }^{20-21}$

Embora a região Sul, assim como as regiões Centro-Oeste e Sudeste, tenha apresentado a tendência temporal estável no período analisado, seu coeficiente médio foi maior, indicando que essa região possui incidência maior da doença. Parece provável que esse resultado esteja relacionado ao índice de desenvolvimento humano (IDH) da região, o qual pode favorecer maior acesso a serviços de saúde e diagnóstico. Evidências apontam altos coeficientes de AIDS em locais com melhores condições de vida e a região Sul reúne os melhores índices do país nas dimensões da saúde, educação e renda. ${ }^{6,22}$

Em relação às características da população estudada, observa-se predominância de casos na faixa etária de 50 a 59 anos. Possivelmente, esse achado se deve ao fato de que essas pessoas podem ter se infectado em faixas etárias anteriores, não realizaram testes diagnósticos para HIV e/ou tiveram diagnóstico tardio da infecção. ${ }^{23-24}$ Esse resultado indica que, para esses indivíduos, 
o envelhecimento virá com todas as consequências de viver com uma condição crônica que acarreta diversas alterações físicas, psicológicas e sociais. ${ }^{4}$ Além disso, o envelhecimento com AIDS traz aumento de custos significativo aos serviços de saúde devido as chances aumentadas dessas pessoas desenvolverem multimorbidades, infecções oportunistas e necessitarem de cuidados especializados, medicamentos e hospitalizações. ${ }^{4,25-26}$

Assim como neste estudo, outros anteriores corroboram que a transmissão heterossexual foi predominante em pessoas com 50 anos ou mais. ${ }^{14-15,17,27}$ A predominância de transmissão do HIV por via sexual nos casos analisados é um achado já esperado, pois segue um padrão de comportamento e ratifica a transmissão sexual como predominante no Brasil. ${ }^{2}$ Esse resultado reforça ainda que pessoas de meia-idade e idosas têm mantido vida sexual ativa associada a sexo desprotegido e devem ser incluídas nas ações de prevenção de HIV.5,28

Outro resultado interessante encontrado nesta pesquisa e que mostra a dinamicidade da epidemia pelo HIV é que inicialmente ela era prevalente em homens, depois houve um período de feminização da infecção e, recentemente, há predominância da infecção no sexo masculino. ${ }^{3}$ Provavelmente, a doença voltou a crescer nos homens porque, de maneira geral, eles são mais resistentes a cuidar de sua saúde e menos propensos a procurar serviços de saúde para prevenção. ${ }^{29}$

O predomínio de casos com baixa escolaridade, variável representativa da condição socioeconômica da população, é um resultado semelhante ao estudo realizado no estado da Paraíba $^{13}$ e outro desenvolvido em Botsuana com pessoas de 50 anos ou mais com HIV. ${ }^{11}$ É provável que isso esteja relacionado ao fato de que Brasil e Botsuana são países em desenvolvimento, cuja população apresenta baixos níveis socioeconômicos. Ademais essa parcela da população advém de um período em que o acesso à formação escolar era incipiente. ${ }^{30}$ Pondera-se ainda que o nível de escolaridade pode influenciar diretamente na prática do autocuidado à saúde, no conhecimento sobre a doença e na compreensão do tratamento. ${ }^{6}$ 
Tendência temporal da incidência da AIDS em pessoas com 50 anos ou mais no Brasil | 12

Apesar de este estudo permitir conhecer a distribuição temporal da AIDS em pessoas com 50 anos ou mais, o mesmo apresenta algumas limitações. Em decorrência de ser uma doença que apresenta sinais e sintomas que se confundem com alterações do processo de envelhecimento, pode haver subnotificações e diagnósticos tardios. Sinaliza-se como outra limitação a utilização de base de dados secundária, com informações limitadas às fichas de notificação, o que não permite aos pesquisadores controlar aspectos relativos à completude dos dados. Diante disso, recomenda-se aos serviços de saúde a realização de educação permanente sobre notificação de agravos em relação ao preenchimento das notificações, digitação e registro das informações.

Outros estudos devem ser desenvolvidos com a temática, no que diz respeito ao envelhecimento com HIV, exercício da sexualidade e prevenção de Infecções Sexualmente Transmissíveis (IST) e AIDS em pessoas de meia-idade e idosas. Recomenda-se analisar a distribuição ao longo do tempo do HIV nessa parcela da população, uma vez que a infecção passou a ser um agravo de notificação compulsória em todo o território nacional somente em 2014.

\section{Conclusão}

Os resultados deste estudo mostraram estabilidade da incidência de AIDS no Brasil e nas regiões Sudeste, Sul e Centro-Oeste, como também aumento da incidência de AIDS nessa população nas regiões Norte e Nordeste. Além disso, verificou-se que a faixa etária de 50 a 59 anos, sexo masculino, ensino fundamental incompleto, raça/cor branca e transmissão heterossexual foi o perfil com maior frequência nos casos analisados.

Esses achados indicam que a AIDS em pessoas mais velhas é relevante no contexto epidemiológico, apontando para a necessidade de incluir esse grupo nas ações de prevenção e cuidado realizadas pelos serviços de saúde em todo o país. Além de contribuir também para organização do cuidado e gestão do HIV na Rede de Atenção à Saúde das PVHIV, dentre elas as que estão nas faixas etárias elencadas no estudo. Recomenda-se que haja reconfiguração das 
práticas profissionais no âmbito da sexualidade das pessoas que estão envelhecendo e implementação de ações de educação em saúde sobre prática sexual segura e vulnerabilidade às IST/AIDS para esse grupo específico.

\section{Referências}

1. Joint United Nations Programme on HIV/AIDS (UNAIDS). Global AIDS update: Fact sheet [Internet]. Geneva: UNAIDS; 2019 [cited 2021 July 08]. (UNAIDS Global statistics, 6). Available from: https://unaids.org.br/wp-content/uploads/2019/11/2019_UNAIDS_WAD2019_FactSheet.pdf

2. Ministério da Saúde (BR), Secretaria de Vigilância em Saúde, Departamento de Vigilância, Prevenção e Controle das Infecções Sexualmente Transmissíveis. Boletim Epidemiológico de HIV/Aids 2019 [Internet]. Brasília (DF): Ministério da Saúde; 2019 [acesso em 2021 jul 08]. Disponível em: http://www.aids.gov.br/pt-br/pub/2019/boletim-epidemiologico-de-hivaids-2019

3. Grangeiro A, Castanheira ER, Nemes MIB. A re-emergência da epidemia de aids no Brasil: desafios e perspectivas para o seu enfrentamento. Interface. 2015;19(52):5-6. doi: 10.1590/1807-57622015.0038

4. Autenrieth CS, Beck EJ, Stelzle D, Mallouris C, Mahy M, Ghys P. Global and regional trends of people living with HIV aged 50 and over: estimates and projections for 2000-2020. PLoS ONE. 2018;13(11):e0207005. doi: 10.1371/journal.pone.0207005

5. Aguiar RB, Leal MCC, Marques APO, Torres KMS, Tavares MTDB. Idosos vivendo com HIV comportamento e conhecimento sobre sexualidade: revisão integrativa. Ciênc Saúde Colet. 2020;25(2):575-84. doi: 10.1590/1413-81232020252.12052018

6. Castro SS, Scatena LM, Miranzi A, Miranzi Neto A, Nunes AA. Tendência temporal dos casos de HIV/aids no estado de Minas Gerais, 2007 a 2016. Epidemiol Serv Saúde. 2020;29(1):e2018387. doi: $10.5123 / \mathrm{s} 1679-49742020000100016$

7. Huang MB, Ye L, Liang BY, Ning CY, Roth WW, Huang JG, et al. Characterizing the HIV/AIDS epidemic in the United States and China. Int J Environ Res Public Health. 2015;13(1):ijerph13010030. doi: 10.3390/ijerph13010030

8. Lima MS, Firmo AAM, Martins-Melo FR. Trends in AIDS-related mortality among people aged 60 years and older in Brazil: a nationwide population-based study. AIDS Care. 2016;28(12):1533-40. doi: 10.1080/09540121.2016.1191608

9. Paula AA, Pires DF, Alves Filho P, Lemos KRV, Veloso VG, Grinsztejn B, et al. Mortality profiles among people living with HIV/AIDS: comparison between Rio de Janeiro and other federative units between 1999 and 2015. Rev Bras Epidemiol. 2020;23:e200017. doi: 10.1590/1980-549720200017

10. Liu Z, Shi O, Yan Q, Fang Q, Zuo J, Chen Y, et al. Changing epidemiological patterns of HIV and AIDS in China in the post-SARS era identified by the nationwide surveillance system. BMC Infect Dis. 
Tendência temporal da incidência da AIDS em pessoas com 50 anos ou mais no Brasil | 14

2018;18(1):700. doi: 10.1186/s12879-018-3551-5

11. Matlho K, Randell M, Lebelonyane R, Kefas J, Driscoll T, Negin J. HIV prevalence and related behaviours of older people in Botswana - secondary analysis of the Botswana AIDS impact survey (BAIS) IV. Afr J AIDS Res. 2019;18(1):18-26. doi: 10.2989/16085906.2018.1552162

12. Maia DAC, Zanin L, Silva ASF, Ambrosano GMB, Flório FM. Notificação de casos de HIV/AIDS em idosos no estado do Ceará: série histórica entre os anos de 2005 a 2014. Rev Bras Geriatr Gerontol. 2018;21(5):542-52. doi: 10.1590/1981-22562018021.180041

13. Nogueira JA, Silva AO, Sá LR, Almeida AS, Monroe AA, Villa TCS. Síndrome da imunodeficiência adquirida em adultos com 50 anos e mais: características, tendência e difusão espacial do risco. Rev Latinoam Enferm. 2014;22(3):355-63. doi: 10.1590/0104-1169.3327.2424

14. Oliveira MLC, Paz LC, Melo GF. Dez anos de epidemia do HIV-AIDS em maiores de 60 anos no Distrito Federal - Brasil. Rev Bras Epidemiol. 2013;16(1):30-9. doi: 10.1590/S1415-790X2013000100003

15. Silva MM, Vasconcelos ALR, Ribeiro LKNP. Caracterização epidemiológica dos casos de AIDS em pessoas com 60 anos ou mais, Pernambuco, Brasil, 1998 a 2008. Cad Saúde Pública. 2013;29(10):2131-5. doi: 10.1590/0102-311X00161112

16. Vieira GD, Alves TC, Sousa CM. Análise dos dados epidemiológicos da aids em idosos no estado de rondônia, amazônia ocidental. DST J Bras Doenças Sex Transm. 2012;24(1):49-52. doi: 10.5533/2177-8264201224112

17. Carvalho LMF, Câmara FP. Epidemiological aspects of acquired immunodeficiency syndrome in older Brazilians: a comparative approach. Braz J Infect Dis. 2012;16(1):34-7. doi: 10.1590/S141386702012000100006

18. Instituto Brasileiro de Geografia e Estatística (IBGE). Mudança demográfica no Brasil no início do século XXI - subsídios para as projeções da população [Internet]. Rio de Janeiro: IBGE; 2015 [acesso em 2021 jul 08]. (Estudos e análises, informação demográfica e socioeconômica, 3). Disponível em: https://biblioteca.ibge.gov.br/visualizacao/livros/liv93322.pdf

19. Teixeira MG, Costa MCN, Paixão ES, Carmo EH, Barreto FR, Penna GO. Conquistas do SUS no enfrentamento das doenças transmissíveis. Ciênc Saúde Colet. 2018;23(6):1819-28. doi: 10.1590/141381232018236.08402018

20. Maia DAB, Frias PG, Assunção RS, Vidal SA, Vanderlei LCM. Evaluation of the implantation of the notifiable diseases information system in Pernambuco State, Brazil, 2014. Epidemiol Serv Saúde. 2019;28(1):e2018187. doi: 10.5123/s1679-49742019000100002

21. Carvalho BR, Ferreira JBB, Fausto MCR, Forster AC. Avaliação do acesso às unidades de atenção primária em municípios brasileiros de pequeno porte. Cad Saúde Colet. 2018;26(4):462-9. doi: 10.1590/1414-462x201800040471

22. Paiva SS, Pedrosa NL, Galvão MTG. Spatial analysis of AIDS and the social determinants of health. Rev Bras Epidemiol. 2019;22:e190032. doi: 10.1590/1980-549720190032 
23. Alencar RA, Ciosak SI. AIDS in the elderly: reasons that lead to late diagnosis. Rev Bras Enferm. 2016;69(6):1076-81. doi: 10.1590/0034-7167-2016-0370

24. Bastos LM, Tolentino JMS, Frota MAO, Tomaz WC, Fialho MLS, Batista ACB, et al. Avaliação do nível de conhecimento em relação à Aids e sífilis por idosos do interior cearense, Brasil. Ciênc Saúde Colet. 2018;23(8):2495-502. doi: 10.1590/1413-81232018238.10072016

25. Guaraldi G, Zona S, Menozzi M, Brothers TD, Carli F, Stentarelli C, et al. Late presentation increases risk and costs of non-infectious comorbidities in people with HIV: an Italian cost impact study. AIDS Res Ther. 2017;14(1):8. doi: 10.1186/s12981-016-0129-4

26. Turrini G, Chan SS, Klein PW, Cohen SM, Dempsey A, Hauck H, et al. Assessing the health status and mortality of older people over 65 with HIV. PLoS One. 2020;15(11):e0241833. doi: 10.1371/journal.pone.0241833

27. Tavoschi L, Dias JG, Pharris A; EU/EEA HIV Surveillance Network. New HIV diagnoses among adults aged 50 years or older in 31 European countries, 2004-15: an analysis of surveillance data. Lancet HIV. 2017;4(11):e514-21. doi: 10.1016/S2352-3018(17)30155-8

28. Andrade J, Ayres JA, Alencar RA, Duarte MTC, Parada CMGL. Vulnerability of the elderly to sexually transmitted infections. Acta Paul Enferm. 2017;30(1):8-15. doi: 10.1590/1982-0194201700003

29. Lemos AP, Ribeiro C, Fernandes J, Bernardes K, Fernandes R. Saúde do homem: os motivos da procura dos homens pelos serviços de saúde. Rev Enferm UFPE On Line [Internet]. 2017 [cited 2021 July 08];11(Suppl 11):4546-53. Available from: https://periodicos.ufpe.br/revistas/revistaenfermagem/article/view/231205

30. Silva PAB, Santos FC, Soares SM, Silva LB. Sociodemographic and clinical profile of elderly persons accompanied by Family Health teams under the gender perspective. Rev Pesq Cuid Fundam. 2018;10(1):97-105. doi: 10.9789/2175-5361.2018.v10i1.97-105

Editora Científica: Tânia Solange Bosi de Souza Magnago

Editora Associada: Graziele de Lima Dalmolin

Fomento / Agradecimento: Coordenação de Aperfeiçoamento de Pessoal de Nível Superior - CAPES

\section{Autor correspondente}

Aenne Zandonadi Rodrigues Santana

E-mail: aennesantana@gmail.com

Endereço: Avenida das Palmeiras, nº 20, Condomínio Rio Jangada, Jardim Imperial, Cuiabá, Mato Grosso, Brasil. CEP: 78075-903 


\section{Contribuições de Autoria}

\section{1 - Aenne Zandonadi Rodrigues Santana}

Concepção ou desenho do estudo/pesquisa, análise e/ou interpretação dos dados, revisão final com participação crítica e intelectual no manuscrito.

\section{2 - Annelita Almeida Oliveira Reiners}

Concepção ou desenho do estudo/pesquisa, análise e/ou interpretação dos dados, revisão final com participação crítica e intelectual no manuscrito.

\section{3 - Rosemeiry Capriata de Souza Azevedo}

Concepção ou desenho do estudo/pesquisa, análise e/ou interpretação dos dados, revisão final com participação crítica e intelectual no manuscrito.

\section{4 - Janderson Diego Pimenta da Silva}

Concepção ou desenho do estudo/pesquisa, análise e/ou interpretação dos dados, revisão final com participação crítica e intelectual no manuscrito.

\section{5 - Amanda Cristina de Souza Andrade}

Concepção ou desenho do estudo/pesquisa, análise e/ou interpretação dos dados, revisão final com participação crítica e intelectual no manuscrito.

\section{6 - Priscila Aguiar Mendes}

Concepção ou desenho do estudo/pesquisa, análise e/ou interpretação dos dados, revisão final com participação crítica e intelectual no manuscrito.

\section{Como citar este Artigo}

Santana AZR, Reiners AAO, Azevedo RCS, Silva JDP, Andrade ACS, Mendes PA. Temporal trend in the incidence of AIDS in people aged 50 and over in Brazil. Rev. Enferm. UFSM. 2021 [Access on: Year Month Day]; vol.11 e59: 116. DOI: https://doi.org/10.5902/2179769263534 